two systems are thus coextensive. Since on $R_{2 n}$ the $g_{n-1}^{1}$ is defined by the generators of one system of the hyperboloid, the envelope of the lines containing the $G_{n-1}$ is the section of the tangent cone to the hyperboloid from the center of projection.

6 . If $\phi_{n}, \phi_{n}^{\prime}, \phi_{n}^{\prime \prime}$ be three cones of order $n$ containing the $n$ bisecants from $(0,0,0,1)$, then, since the $n-1$ remaining edges of intersection with the cone $R_{2 n}$ from the same point lie in a plane, the equation of the defining monoid may be written

$$
w=\frac{x \phi_{n}^{\prime}}{\phi_{n}}=\frac{x \phi_{n}^{\prime \prime}}{\phi_{n}^{\prime}},
$$

from which the equation (1) results. Incidentally, these equations furnish a means for reducing $c_{2 n}$ to $c_{2 n-1}$, namely, the $\infty^{2}$ plane sections of the monoid from a point on $R_{2 n}$, lying on one of the $n-1$ simple edges.

7. If $R_{2 n}$ has also actual double points or cusps, $\phi_{n}$ will not in general pass through them, hence in the plane curves $c_{2 n}$ we can distinguish between projection of actual double points and apparent double points. Actual double points will not always absorb two coincidences in the $[n-2]$ involutions, but when $p<\frac{1}{2}(n-1)(n-2)$, the projection curve can not be written in the form (1).

For other curves on the hyperboloid, the maximum number of basis lines of a net formed by bisecants will not be reached; but when no actual double points occur we may say that the projection curve $c_{n}$ with $p>\frac{1}{6}(n-1)(n-2)$ cannot be birationally transformed into any curve of order less than $n-1$.

CORNell UNIVERSITY, August, 1907.

\title{
NOTE ON CERTAIN INVERSE PROBLEMS IN THE SIMPLEX THEORY OF NUMBERS.
}

BY PROFESSOR R. D. CARMICHAEL.

(Read before the American Mathematical Society, September 5, 1907.)

Legendre* has considered the problem of finding the highest power of a prime $p$ contained in $m !=1 \cdot 2 \cdot 3 \cdots m$. Let $m$ be written in the form

$$
m=a_{0} p^{\alpha}+a_{1} p^{\beta}+a_{2} p^{\gamma}+\cdots,
$$

* A. M. Legendre, Théorie des nombres, 3d ed., I., p. 10. 
where $\alpha, \beta, \gamma, \cdots$ are different positive integers (including zero) and $a_{0}, a_{1}, a_{2}, \ldots$ are the same or different positive integers each less than $p$. Put

$$
a_{0}+a_{1}+a_{2}+\cdots=s .
$$

Then the highest power of $p$ contained in $m !$ is $p^{(m-s) /(p-1)}$; or, in the congruence

$$
m ! \equiv 0\left(\bmod p^{(m-s) /(p-1)}\right),
$$

the modulus gives the highest power of $p$ for which the congruence holds.

The problem first to be considered in this note is to find the solutions of (3) when $p$ and $s$ are known and $m$ is the unknown.*

Suppose a solution

$$
m_{1}=a_{0} p^{\alpha}+a_{1} p^{\beta}+a_{2} p^{\gamma}+\cdots
$$

has been found. Take $\alpha$ to be the least of the numbers $\alpha, \beta, \gamma, \cdots$ and divide equation (4) by $p^{a}$. We obtain

$$
\frac{m_{1}}{p^{\alpha}}=a_{0}+a_{1} p^{\beta-\alpha}+a_{2} p^{\gamma-\alpha}+\cdots=m_{2} \text { say. }
$$

It is clear from the first paragraph that $m_{2}$ is also a solution of (3). Moreover if $m_{2}$ is multiplied by any power of $p$ the resulting number is also a solution of (3), subject to all the conditions which have been imposed. Therefore,

To every solution of (3) there corresponds an infinite number of solutions differing only by factors which are powers of $p$.

We shall define a characteristic solution to be such a one as that given in (5); thus, a characteristic solution of (3) is one for which $m$ is not divisible by $p$.

Now suppose $s$ separated in any way into parts each of which is less than $p$ and let these parts be $a_{0}, a_{1}, a_{2}, \ldots$ Then (1) is a solution of (3) however $\alpha, \beta, \gamma, \cdots$ may be chosen so that

* The modified problem of finding the solutions of the congruence

$$
m ! \equiv 0\left(\bmod p^{v}\right)
$$

subject to the limitation that $p^{v}$ is the highest power of $p$ in $m !$, is very easy. It is clear that the smallest solution is always a multiple of $p$, say $=\mu p$. Then other solutions are $\mu p+1, \mu p+2, \cdots, \mu p+p-1 ;$ moreover these are all the possible solutions. It is to be noticed that a solution of this problem does not always exist. A case in point is $p=3, v=3$; for these values there is no solution of the congruence subject to the imposed conditions. 
they are different positive integers including zero. Hence, when $s>1$ so that this partition is possible, there is an infinite number of solutions for each partition of $s$ as above. When $s=1$ it is clear that the solutions are $m=1, p, p^{2}, \cdots$. Furthermore it is evident that there can be no solutions except those which are here obtained by aid of the partitions of $s$ into parts each less than $p$.

As a second problem we shall consider the solution of

$$
m ! \equiv 0\left(\bmod p^{m-t}\right),
$$

where $p^{m-t}$ is the highest power of $p$ contained in $m !, m$ still being the unknown. For every solution $m$ we have from the proposition of the first paragraph

$$
m-t=\frac{m-s}{p-1}
$$

whence, if $p \neq 2$, that is, if $p$ is an odd prime,*

$$
m=\left(1+\frac{1}{p-2}\right) t-\frac{s}{p-2}
$$

where $s$ as before equals $a_{0}+a_{1}+a_{2}+\cdots$, the sum of the coefficients of the powers of $p$ when $m$ is expressed in the form of (1). Therefore, since

$$
m<\left(1+\frac{1}{p-2}\right) t,
$$

a definite constant, the number of solutions of this problem is finite.

Now suppose

Then

$$
m=c_{n} p^{n}+c_{n-1} p^{n-1}+\cdots+c_{1} p+c_{0} .
$$

$$
s=c_{n}+c_{n-1}+\cdots+c_{1}+c_{0} .
$$

Hence from (8) we may write

$$
\begin{gathered}
c_{n} p^{n}+c_{n-1} p^{n-1}+\cdots+c_{1} p+c_{0}+\frac{c_{n}+\cdots+c_{1}+c_{0}}{p-2} \\
=\left(1+\frac{1}{p-2}\right) t=r_{\nu} p^{\nu}+\cdots+r_{1} p+r_{0}+\frac{u}{p-2}
\end{gathered}
$$

* If $p=2, t=s$; this problem therefore reduces to the preceding for the case $p=2$. Its solution has already been found. We shall henceforth consider $p$ an odd prime. 
where $m$ and $s$ are replaced by their values above and $r_{v}, \ldots, r_{0}$ are integers each less than $p$ and $u<p-2$. The $r$ 's are uniquely determined from the known value of $\left[1+1 f^{\prime}(p-2)\right]$. Now

$$
\frac{c_{n}+\cdots+c_{0}}{p-2} \equiv \frac{(n+1)(p-1)}{p-2}<p^{n}, \text { if } n>1 .
$$

Therefore in equation (10) $n=\nu$, when $n>1$; then also $c_{n}=r_{v}$. Having obtained these values we may proceed similarly to consider the coefficeints $c_{n-1}, r_{\nu-1}$ and so on. All the possible solutions will be obtained with but little difficulty. The work will be made easy from the fact that the coefficients of like powers of $p$ are the same in the two members of (10) except for small exponents of $p$.

As an example, consider the solution of the problem when $p=11$ and $t=14578$. We have

$$
\left(1+\frac{1}{p-2}\right) t=16197 \frac{\pi}{9}=11^{4}+11^{3}+11^{2}+9 \cdot 11+5+\frac{7}{9} .
$$

Comparing with (10) we have $n=4, c_{4}=1$. Then without difficulty we may further show that $c_{3}=1$ and $c_{2}=1$. Substituting in (10) and reducing we have

$$
c_{1} \cdot 11+c_{0}+\frac{c_{1}+c_{0}}{9}=9 \cdot 11+5+\frac{4}{9} .
$$

From this equation it is readily shown that $c_{1}>8$ and $<10$; and therefore its only possible value is $c_{1}=9$. This gives $c_{0}=4$. Therefore,

$$
m=11^{4}+11^{3}+11^{2}+9 \cdot 11+4=16196 .
$$

There is nothing in the preceding discussion to show that a solution always exists for this problem. An examination of the product of the natural numbers in order, reference being had to how often any prime has entered up to any number $m$, will easily lead to the discovery that the consecutive values of $t$ contain every integer beginning with 1 and that some integers will be repeated in the series of values of $t$. Hence, there always exists at least one solution; and in some cases there is more than one solution.

Anniston, Ala., May, $190 \%$. 